Research Article

\title{
A Potential Failure Mode and Effect Analysis Method of Electromagnet Based on Intuitionistic Fuzzy Number in Manufacturing Systems
}

\author{
Jihong Pang, ${ }^{1,2}$ Jinkun Dai, ${ }^{2}$ and Faqun Qi $\mathbb{D}^{2}$ \\ ${ }^{1}$ College of Business, Shaoxing University, Shaoxing 312000, China \\ ${ }^{2}$ College of Mechanical and Electrical Engineering, Wenzhou University, Wenzhou 325035, China \\ Correspondence should be addressed to Faqun Qi; qifaqunqq@163.com
}

Received 15 May 2021; Accepted 5 August 2021; Published 23 August 2021

Academic Editor: Francesco Tornabene

Copyright (c) 2021 Jihong Pang et al. This is an open access article distributed under the Creative Commons Attribution License, which permits unrestricted use, distribution, and reproduction in any medium, provided the original work is properly cited.

Failure mode and effect analysis (FMEA) is a systematic activity in the stage of product design and process design. However, the traditional FMEA has some shortcomings in practical application, such as too many evaluation languages, uncertain weights of influencing factors, and uncertain weights of evaluation members. This paper presents an FMEA evaluation method in manufacturing system based on similarity measure, nonlinear programming model, and intuitionistic fuzzy number (IFN). Firstly, the IFN is used to evaluate failure mode, which overcomes the defect of traditional FMEA evaluation value. Secondly, the weight of failure evaluation team members is solved according to the concept of similarity measure to make up for the blank of evaluation members' weight aiming at the shortage of unknown weight. Then, the definition of consensus measure is introduced to make the evaluators reach a consensus, and the weights of influencing factors of failure modes (FMs) are calculated. Finally, the weights of evaluators and influencing factors are calculated by IFN algorithm and score function, and the score value of each FM is obtained to rank instead of risk priority number (RPN). The objectivity and practicability of the new method are verified by the example of failure mode for an attractive electromagnet manufacturing system.

\section{Introduction}

FMEA is a systematic activity in product design stage and process design stage. The subsystems and parts of FMEA are analyzed one by one to identify all potential FMs and their possible consequences, so as to take necessary measures in advance to improve the quality and reliability of products. FMEA was originally carried out in the product design phase of the aerospace industry in the 1960s to help improve safety and reliability [1]. In addition, FMEA enables an organization to proactively prevent failure, not just respond to it [2]. FMEA is also a simple method to solve the complex failure mode evaluation process [3]. Because of its simple and easy operation, FMEA is widely used in transportation, energy, medical, and supply chain transportation $[4,5]$.

On the other hand, the traditional FMEA process is used to evaluate the FMs of products or systems by team members. The severity, occurrence, and detection (S, O, D) of FMs according to their own professional knowledge are calculated by the scores of each failure mode [6]. Although FMEA is widely used, it has some inherent defects. To ensure the objectivity of FMEA, we must ensure that the evaluation language of failure mode should conform to the actual situation, so as to make a more comprehensive and specific description of failure mode. However, the traditional FMEA uses natural language to carry out evaluation, which does not conform to the psychological changes of the evaluation members in reality [7]. Secondly, the weight of factors is also the key to affect the final evaluation results [8]. For example, the weights of influencing factors are evenly distributed by traditional FMEA, which will lead to great difference between the calculation results and the actual situation [9]. In the actual production, the evaluation members in different working positions have different understanding of the 
definition and importance of failure; it is necessary to accurately calculate the weight of each evaluation member in the evaluation team to determine the failure mode sequencing $[10,11]$.

In order to improve the shortcomings of the traditional FMEA, many scholars and researchers have carried out more rigorous research on this method and made it more objective and true to reflect the impact of failure. So, further research in this area would be needed. A comprehensive risk ranking method to improve the performance of FMEA was developed by using interval-valued intuitionistic fuzzy sets (IVIFSs) and multiattribute boundary approximation area comparison (MABAC). In addition, a linear programming model to obtain the optimal weight of risk factors was established under the condition of incomplete prior knowledge of weight information [12]. A new integrated fuzzy intelligent FMEA framework was proposed. The framework adopts the combination of fuzzy set theory, analytic hierarchy process (AHP), and data envelopment analysis (DEA) to deal with uncertainty and improve the reliability of risk assessment. All of these are achieved by employing a heterogeneous expert group. RPN, time, and cost are used as indicators to determine the efficiency of FMEA mode and determine the appropriate priority and corrective measures [13]. An interactive method to deal with the possible uncertainties in the evaluation process was proposed by using the fuzzy set theory. In order to deal with the weight of subjective and objective uncertainties, the AHP and entropy weight method were adopted [14]. A new method combining evidence theory, intuitive fuzzy set (IFS), and belief entropy has been proposed to determine the weight of team members to analyze FMs [15]. A hybrid FMEA framework has been developed which integrates the Portuguese abbreviation for interactive and multicriteria decision method with the Choquet integration method. Under this framework, the uncertainty in risk assessment is described by generalized trap type fuzzy numbers [16]. A consensus-based FMEA group decision-making framework was proposed. The purpose of this framework is to classify FMs into several ordinal risk classes. Assuming that FMEA participants provide their preferences in a linguistic manner using the possibility hesitant fuzzy linguistic information, a consensus driven method is proposed to generate the weight of risk factors in the FMEA framework. On this basis, the consensus rules based on optimization guided by the minimum adjustment distance strategy are calculated, and an interaction model for consensus is developed to generate consensus failure mode risk classes [17]. A triangular distribution-based basic probability assignment (TDBPA) method based on triangular distribution was presented within the framework of Dempster-Shafer evidence theory, the conflict risk levels assessed by different experts. The modified RPN model based on fusion assessment could calculate the ranking of FMs [18]. A new comprehensive multicriteria decision-making (MCDM) method and fuzzy multiattribute ideal real comparative analysis (FMAIRCA) were proposed. It combines the first method to calculate the fuzzy relative importance between risk factors by using quantitative method [19]. In order to improve the performance of classical FMEA, the weights of influencing factors are determined by using the fuzzy optimization method, and a fuzzy VIKOR approach was to evaluate the security policies and analyze the content of five press agencies in expert decision making [20].

In this paper, the concept of similarity measurement is used to solve the weights of evaluation members and makes up for the blank of obtaining weights of evaluation members on the basis of existing research further to improve the FMEA. In the same time, the definition of consensus measurement is introduced to reach consensus on the evaluation of the evaluators, and the weights of influencing factors are solved by using the approved method. The remainder of this paper is arranged as follows: in Section 2, a decision process of electromagnet FMEA is presented. Section 3 describes the IFN evaluation of failure mode with a form of influencing factors. In Section 4, the weight of evaluation members and influencing factors is determined by comprehensive calculation, and the IFN of FMs is obtained. The scores of each failure mode are obtained and sorted according to the score function. An illustrative example of FMEA of electromagnet is shown in Section 5. The last chapter summarizes the work done in this paper.

\section{Flow Chart of FMEA of Electromagnet}

FMEA methodically breaks down the analysis of complex manufacturing processes of electromagnet into manageable steps. FMEA can be used to identify potential failure modes for electromagnet and determine their impacts during production and fabrication. The flow chart of FMEA of electromagnet is shown in Figure 1.

To achieve the desired effect of this paper, the following key steps are taken. Step 1: determine the target and risk level of the electromagnet product risk analysis, collect the potential failure mode and failure impact, and the evaluation team evaluates the failure mode by using the IFN. Step 2: according to the incomplete weight information in FMEA, the similarity measure is used to determine the weight of evaluation team members. Step 3: for the influencing factors of failure, the evaluation members use IFNs for comparative evaluation and form a comparison matrix. In order to find the optimal solution of the influencing factors, the exact weight of the influencing factors is derived from the preference relation of the comparison matrix. Step 4: after the weight information in FMEA is all determined, simple intuitionistic fuzzy weighted geometry (SIFWG) is used to aggregate the evaluation information and weight information, and the score of each failure mode is calculated according to the definition of intuitionistic fuzzy score function, and the order is made.

\section{Evaluation of IFNs on FMs and Influencing Factors}

In 1965, Zadeh defined the mathematical meaning of IFSs for the first time [21] and opened a new chapter of fuzzy mathematics. In 1986, Atanassov popularized fuzzy sets, and 


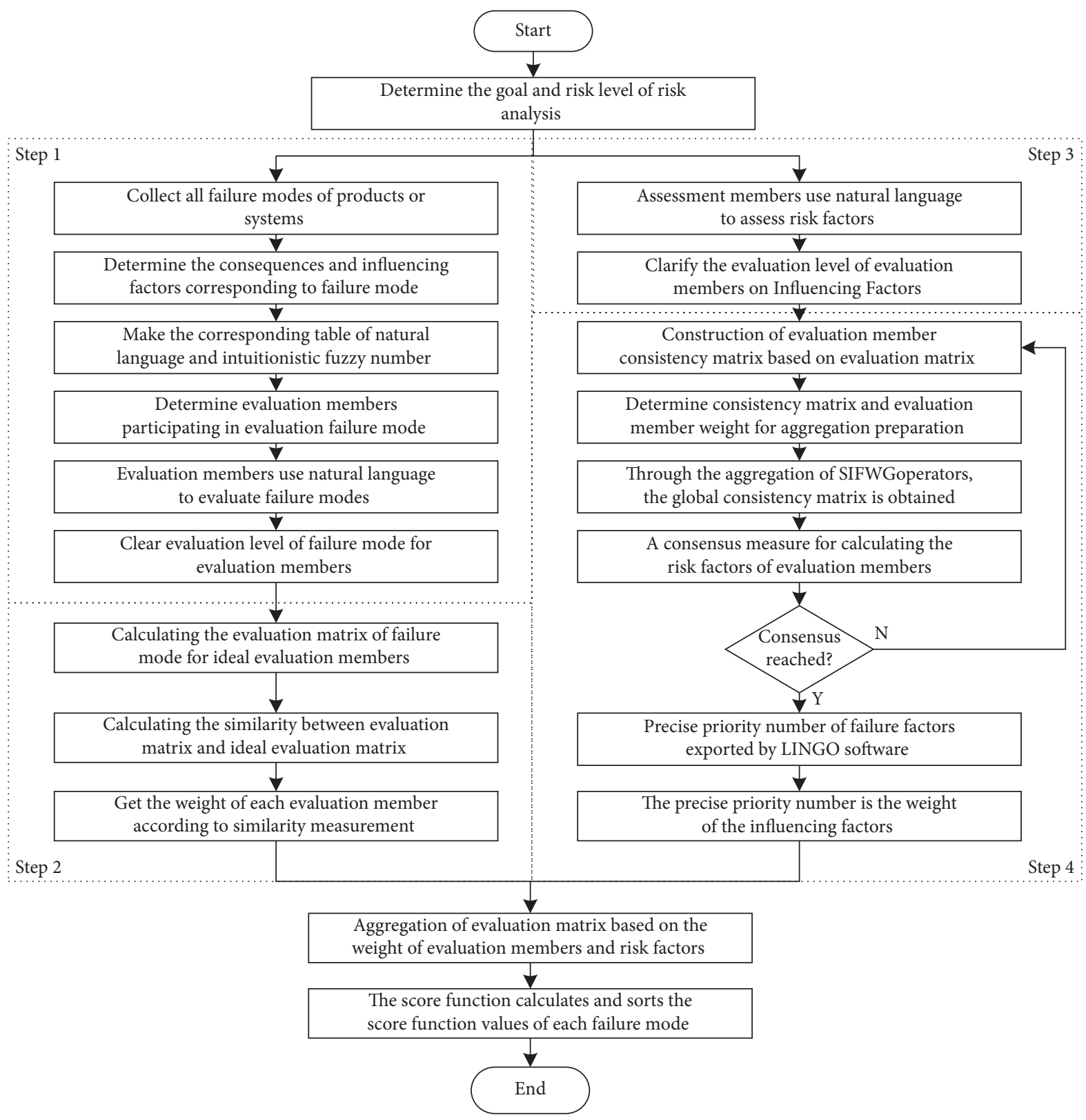

FIgURE 1: Flow chart of FMEA of electromagnet.

it was also the first time to express the relationship between an element and a specific set with the three indexes of membership degree, nonmembership degree, and hesitation, and he put forward the concept of IFSs [22]. Let the set $X$ be a universe of discourse, the fuzzy set $F$ on $X$ is represented by membership degree $\mu_{F}, \mu_{F}: X \longrightarrow[0,1]$, and then the membership degree of $x$ in the set $\mathrm{F}$ is denoted by $\mu_{F}(x)$. IFS is an object that has the following form:

$$
A=\left\{x, \mu_{A}(x), v_{A}(x) \mid x \in X\right\},
$$

where $\mu_{A}(x), v_{A}(x)$ means the membership degree and nonmembership degree of the element $x \in X$ to $A$, respectively, with the condition that for all $x \in X$, then

$$
\begin{aligned}
& 0 \leq \mu_{A}(x) \leq 1, \\
& 0 \leq \nu_{A}(x) \leq 1, \\
& 0 \leq \nu_{A}(x)+\mu_{A}(x) \leq 1 .
\end{aligned}
$$

3.1. Basic Operation Law of IFN. Let $a=\left(\mu_{a}, v_{a}\right)$ be IFN, then the basic operation law of IFN is displayed as follows [23]:

$$
\begin{aligned}
& \lambda a=\left(1-\left(1-\mu_{a}\right)^{\lambda}, \nu_{a}^{\lambda}\right), \\
& a^{\lambda}=\left(\mu_{a}^{\lambda}, 1-\left(1-\nu_{a}\right)^{\lambda}\right),
\end{aligned}
$$




$$
\begin{aligned}
& \stackrel{\substack{j=1 \\
\oplus}}{n} a_{j}=\left(\begin{array}{cc}
\substack{j=1 \\
n} & \substack{j=1 \\
\prod} \\
& \stackrel{n}{n} \nu_{a_{j}}
\end{array}\right),
\end{aligned}
$$

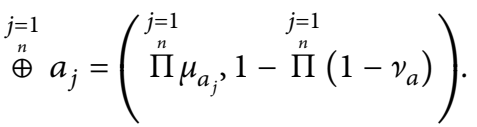

3.2. Ranking Rules of IFNs. Let $a=\left(\mu_{a}, v_{a}\right)$ be IFN; its score function $S(a)$ and accuracy function $H(a)$ are expressed, respectively, by the following formulas:

$$
\begin{aligned}
& S(a)=\left|\mu_{a}-v_{a}\right|, \\
& H(a)=\mu_{a}+v_{a} .
\end{aligned}
$$

Let $a_{i}=\left(\mu_{a_{i}}, v_{a_{i}}\right)$ and $a_{j}=\left(\mu_{a_{j}}, v_{a_{j}}\right)$ be two IFNs, then the procedure of comparing the magnitude of two IFNs is introduced as follows:

$$
\text { if } S\left(a_{i}\right)<S\left(a_{j}\right) \text {, then } a_{i}<a_{j} \text {. }
$$

If $S\left(a_{i}\right)=S\left(a_{j}\right)$, then we can get the following conclusions:

$$
\begin{aligned}
& \text { If } H\left(a_{i}\right)<H\left(a_{j}\right) \text {, then } a_{i}<a_{j} . \\
& \text { If } H\left(a_{i}\right)=H\left(a_{j}\right) \text {, then } a_{i}=a_{j} .
\end{aligned}
$$

Because the similarity function is improved on the basis of score function, all similarity functions $L(a)$ conform to the rules of score function.

\section{Failure Mode and Effect Analysis}

FMEA is a comprehensive calculation of decision information of failure mode by comprehensive decision personnel. The total proportion of influencing factors by each evaluation member can directly affect the ranking of final failure mode. Therefore, the weights of objective evaluation members and influencing factors are determined before the final comprehensive calculation.

4.1. Determination of the Weight of Evaluation Members. Considering all possible options, we select the risk assessment

matrices $X^{k}=\left[x_{i j}^{k}\right]_{m \times n}=\left[\mu_{i j}^{k}, v_{i j}^{k}\right]_{m \times n}$, where $k=1, \ldots, l$, and adopt many ways to estimate the evaluation model. Next, the ideal assessment of the failure mode can be the average of a single risk assessment matrix, and the expression of ideal matrix is shown as follows [24]:

$$
X^{*}=\left[x_{i j}^{*}\right]_{m \times n}=\left[\mu_{i j}^{*}, v_{i j}^{*}\right]_{m \times n},
$$

where $x^{*}=1 / l x_{i j}^{k}, k=1, \ldots, 1, \quad$ and $\quad \mu_{i j}^{*}=1-\prod_{k=1}^{l}$ $\left(1-\mu_{i j}^{k}\right)^{1 / l}, v_{i j}^{*}=\prod_{k=1}^{l}\left(v_{i j}^{k}\right)^{1 / l}, i=1, \ldots, m, j=1, \ldots, n$.

Let $a_{1}=\left(\mu_{a_{1}}, v_{a_{1}}\right)$ and $a_{2}=\left(\mu_{a_{2}}, v_{a_{2}}\right)$ be two IFNs, then the interval-valued intuitionistic fuzzy Euclidean distance is calculated as

$$
d\left(a_{1}, a_{2}\right)=\sqrt{\frac{1}{2}\left(\left(\mu_{1}-\mu_{2}\right)^{2}+\left(\nu_{1}-v_{2}\right)^{2}\right)} .
$$

Let $X^{1}=\left(x_{i j}^{1}\right)_{m \times n}=\left(\mu_{i j}^{1}, v_{i j}^{1}\right), X^{2}=\left(x_{i j}^{2}\right)_{m \times n}=\left(\mu_{i j}^{2}, v_{i j}^{2}\right)$ be two interval-valued intuitionistic fuzzy matrixes, then the similarity measure of the two matrixes is defined as

$$
\operatorname{sim}\left(X^{1}, X^{2}\right)=\frac{\sum_{i=1}^{m} \sum_{j=1}^{n} d\left(x_{i j}^{1}, x_{i j}^{2 c}\right)}{\sum_{i=1}^{m} \sum_{j=1}^{n}\left(d\left(x_{i j}^{1}, x_{i j}^{2 c}\right)+d\left(x_{i j}^{1}, x_{i j}^{2}\right)\right)},
$$

where $d$ is the Euclidean distance, $x_{i j}^{2 c}=\left(v_{i j}^{2}, \mu_{i j}^{2}\right)$, $0 \leq \operatorname{sim}\left(X^{1}, X^{2}\right) \leq 1$, when $X^{1}=X^{2}, \operatorname{sim}\left(X^{1}, X^{2}\right)=1$.

Next, the similarity between the evaluation results of each evaluator and the ideal evaluation results is obtained

$$
\operatorname{sim}\left(X_{k}, X^{*}\right)=\frac{\sum_{i=1}^{m} \sum_{j=1}^{n} d\left(x_{i j}^{k}, x_{i j}^{* c}\right)}{\sum_{i=1}^{m} \sum_{j=1}^{n}\left(d\left(x_{i j}^{k}, x_{i j}^{* c}\right)+d\left(x_{i j}^{k}, x_{i j}^{*}\right)\right)},
$$

where $X^{* c}=\left[x_{i j}^{* c}\right]_{m \times n}=\left[v_{i j}^{*}, \mu_{i j}^{*}\right]_{m \times n}$.

At last, the weights of the FMEA team members are determined as follows:

$$
\lambda_{k}=\frac{\operatorname{sim}\left(X_{k}, X^{*}\right)}{\sum_{k=1}^{l} \operatorname{sim}\left(X_{k}, X^{*}\right)} .
$$

\subsection{Determination of the Weight of Influencing Factors}

4.2.1. Construction of Perfect Product Consistency Matrix. In the traditional AHP, the consistency ratio (CR) is used to measure the consistency of the product relation. In this paper, the intuitionistic fuzzy matrix of $R=\left(r_{i k}\right)_{m \times m}=$ $\left(\mu_{i k}, v_{i k}\right)$ is constructed into a perfect product consistent intuitionistic fuzzy matrix $R^{p}=\left(r_{i k}^{p}\right)_{m \times m}$ by using the following algorithm:

(1) If $k>i+1, r_{i k}^{p}=\left(\mu_{i k}^{p}, v_{i k}^{p}\right)$, where

$$
\mu_{i k}^{p}=\frac{\sqrt[k-i-1]{\prod^{k=i+1}} \mu_{i t} \mu_{t k}}{\sqrt[k-i-1]{\prod^{\frac{t=i+1}{k-1} \mu_{i t} \mu_{t k}}+\sqrt[k-i-1]{\prod^{t=i+1}}\left(1-\mu_{i t}\right)\left(1-\mu_{t k}\right)}}
$$

$$
v_{i k}^{p}=\frac{\sqrt[k-i-1]{\prod_{\substack{t=i+1 \\ k-1}} v_{i t} \nu_{t k}}}{\sqrt[k-i-1]{\prod_{k=i+1}^{k-1} v_{i t} \nu_{t k}}+\sqrt[k-i-1]{\prod_{t=i+1}^{k-1}\left(1-v_{i t}\right)\left(1-v_{t k}\right)}} .
$$

(2) If

$$
k=i+1, \quad r_{i k}^{p}=r_{i k}
$$

(3) If

$$
k<i+1, \quad r_{i k}^{p}=\left(v_{i k}^{p}, \mu_{i k}^{p}\right) .
$$


4.2.2. Determination and Iterative Modification of Consistency Measure. Generally, the level of evaluation members is basically the same, and the evaluation results obtained should coincide. However, the inconsistency of evaluation results cannot be avoided in real life. The process of group consensus is to find the final solution accepted by the evaluated personnel. The following gives an algorithm to reach consensus.

Step 1: calculating the consensus degree of evaluation members,

$$
C_{l}=1-\frac{\sum_{1 \leq i \leq j \leq m}^{m}\left(\left|\mu_{i j}^{p}-\mu_{i j}^{*}\right|+\left|v_{i j}^{p}-v_{i j}^{*}\right|+\left|\pi_{i j}^{p}-\pi_{i j}^{*}\right|\right)}{(m-1)(m-2)} .
$$

If the consensus degree reaches the critical value $\gamma$ of group consensus, the result of SIFWG will be output; otherwise, turn to step 2.

Step 2: revising according to the formula,

$$
\mu_{i j}^{l p}=\left(\mu_{i j}^{l p}\right)^{\xi} \times\left(\mu_{i j}^{*}\right)^{1-\xi}, v_{i j}^{l p}=\left(v_{i j}^{l p}\right)^{\xi} \times\left(v_{i j}^{*}\right)^{1-\xi},
$$

where $R^{l p}=\left(r_{i k}^{l p}\right)_{m \times n}=\left(\mu_{i j}^{l p}, v_{i j}^{l p}\right)_{m \times n}$ is the perfect product consistent intuitionistic fuzzy matrix of evaluator $l$.

Step 3: calculating the consensus degree.

In this paper, we use the new modified perfect product consistent intuitionistic fuzzy matrix to calculate the consensus degree. If it meets the critical value, the new modified perfect product consistent intuitionistic fuzzy matrix is aggregated with SIFWG operator to form a new aggregation matrix. If it does not meet the critical value, turn to Step 2 until it meets the critical value.

\subsubsection{Aggregation of Evaluation Information and Derivation} of Influencing Factors' Weight. According to abovementioned algorithm, the perfect product consistency intuitionistic fuzzy matrix $R^{p}=\left(r_{i k}^{p}\right)_{m \times n}$ is obtained. For information aggregation, there are many operators that can be used. This paper uses SIFWG operator, because the matrix before aggregation has consistency, and because the after aggregation through SIFWG operator has the same consistency. Then, the matrix $R^{*}=\left(r_{i j}^{*}\right)_{m \times n}=\left(\mu_{i j}^{*}, v_{i j}^{*}\right)$ of SIFWG aggregation is obtained, which is shown in the following:

$$
\mu_{i j}^{*}=\prod_{k=1}^{l}\left(\mu_{i j}^{p}\right)^{\lambda_{k}}, v_{i j}^{*}=\prod_{k=1}^{l}\left(v_{i j}^{p}\right)^{\lambda_{k}}, \quad i=1, \ldots, m, j=1, \ldots, n .
$$

According to the accurate priority derivation method of IFNs, the nonlinear programming model is solved by LINGO software, and the weight of influencing factors is obtained directly [25]. Based on the standard operation of LINGO software, we take the matrix as an example to show the nonlinear programming model:

$$
\begin{gathered}
\min f=\sum_{i=1}^{m-1} \sum_{k=i+1}^{m}\left(\rho_{i k}+\delta_{i k}\right), \\
\operatorname{s.t}\left\{\begin{array}{l}
\frac{\omega_{i}}{\omega_{i}+\omega_{k}}+\rho_{i k}-\mu_{i k} \geq 0, \\
\frac{\omega_{i}}{\omega_{i}+\omega_{k}}-\delta_{i k}+\nu_{i k} \geq 0, \\
\sum_{i=1}^{m} \omega_{i}=1, \omega_{i} \in[0,1], \\
\rho_{i k} \geq 0, \delta_{i k} \geq 0, \rho_{i k} * \delta_{i k}=0 .
\end{array}\right.
\end{gathered}
$$

$\operatorname{Export} \omega=\left(\omega_{s}, \omega_{o}, \omega_{d}\right)$,

where $i=1, \ldots, m-1 ; k=i+1, \ldots, m, \rho_{i k}, \delta_{i k}$ is the relaxation variable, $\mu_{i k}-\rho_{i k} \leq \omega_{i} / \omega_{i}+\omega_{k} \leq 1-v_{i k}+\delta_{i k}$.

4.3. Potential Failure Mode Score and Ranking. In this paper, we present the integrate evaluator and influence factor weights with evaluation information, which is shown as

$$
\mathrm{FM}_{i}=\sum_{k=1}^{l} \lambda_{k}\left(\mu_{i j}^{k}, \mu_{i j}^{k}\right) \omega_{S}+\sum_{k=1}^{l} \lambda_{k}\left(\mu_{i j}^{k}, \mu_{i j}^{k}\right) \omega_{O}+\sum_{k=1}^{l} \lambda_{k}\left(\mu_{i j}^{k}, \mu_{i j}^{k}\right) \omega_{d}
$$

where the evaluation information of $k$ assessor is $\left(\mu_{i j}^{k}, \mu_{i j}^{k}\right) i=1, \ldots, m, j=1, \ldots, n$.

Finally, the IFN $\left(\mu_{\mathrm{FM}}, v_{\mathrm{FM}}\right)$ of failure mode is obtained. According to the score function formula of IFN, the score of each failure mode is sorted.

\section{An Example}

This chapter is divided into two sections. Section 5.1 shows the calculation process and precautions of each part of the new method through an evaluation example of the attractive electromagnet. Section 5.2 illustrates the objectivity and practicability of this method by comparing with the other three methods.

5.1. An Example in Manufacturing Systems. The attractive electromagnet itself has a plunger. When the coil is energized, it will attract the plunger to a certain distance and transmit the movement to other loads requiring mechanical action. The structure is shown in Figure 2. They are widely used in electromagnetic relays, solenoid valves, and other products. When the suction electromagnet works, it not only needs to complete the specified movement but also needs to be in the position. The iron reaches the load area, so the consequence of its failure mode is very serious. This paper takes the FMEA of suction electromagnet in the early stage of production as an example to verify the practicability and objectivity of this paper. Since the evaluation information of evaluation members in this paper is in natural language, it is necessary to convert natural language into IFN for 


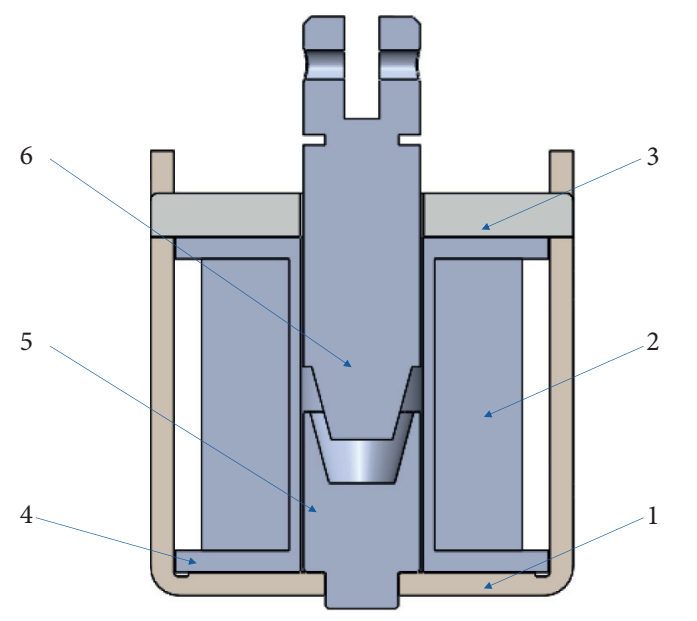

FIGURE 2: Schematic diagram of suction electromagnet structure.

calculation. The corresponding table of natural language and IFN is shown as Table 1, and the main FMs are shown in Table 2.

The company organized three evaluators to evaluate the $S, O$, and $D$ of the fault in natural language mode according to the evaluation criteria of influencing factors in Tables 3-5.

Also, the results of the computations are shown in Table 6 .

Then, according to the corresponding table of natural language and IFN in Table 1, the evaluation table in natural language is transformed into the form of IFN; calculated results are given in Table 7.

According to formulas (14)-(20), the weight of evaluation members is determined, $\lambda_{1}=0.316, \lambda_{2}=0.34$, and $\lambda_{3}=0.344$.

The comparison matrix is obtained by comparing the influence factors of the evaluation members, as shown in Table 8.

The natural language in Table 8 is transformed into IFN according to the corresponding table of natural language and IFN, and then according to algorithm 1, the perfect product consistent intuitionistic fuzzy matrix is constructed, as shown in Table 9.

According to the SIFWG aggregation operator of formula (23), the weight of all evaluators, $\lambda$, has been obtained, $\lambda_{1}=0.316, \lambda_{2}=0.34$, and $\lambda_{3}=0.344$, for example, the aggregation result of membership degree of influencing factor $S$ compared with that of influencing factor $O$ $=0.7^{0.316} * 0.8^{0.34} * 0.8^{0.344}=0.7669$. The aggregation result of nonmembership degree $=0.2^{0.316} * 0.1^{0.34} * 0.1^{0.344}=$ 0.1245 . Similarly, other polymerization results are shown in Table 10.

According to formula (21), the consensus degree of each evaluator is $C_{1}=0.5722, C_{2}=0.7422, C_{3}=0.8537$. If the critical value of consensus degree $C$ is set to 0.5 , then all three evaluators meet the critical value, and it is generally considered that consensus can be reached only when the critical value $C=0.85$. Then, it is modified according to formula (22), where the iterative operation is taken as $\xi=0.5$. After three iterations, the consensus reached is
TABLE 1: Table of natural language and IFNs for failure mode assessment.

\begin{tabular}{lc}
\hline Natural language & IFN \\
\hline Extremely low (EL) & $(0.10,0.90)$ \\
Very low (VL) & $(0.10,0.75)$ \\
Low (L) & $(0.25,0.60)$ \\
Medium low (ML) & $(0.40,0.50)$ \\
Medium (M) & $(0.50,0.50)$ \\
Medium high (MH) & $(0.60,0.30)$ \\
High (H) & $(0.70,0.20)$ \\
Very high (VH) & $(0.80,0.10)$ \\
Extremely high (EH) & $(0.90,0.10)$ \\
Definitely high (DH) & $(1.00,0.00)$ \\
\hline
\end{tabular}

$C_{1}^{\prime}=0.8682, C_{2}^{\prime}=0.8528, C_{3}^{\prime}=0.9544$. When consensus is reached, the matrix aggregated by SIFWG operators is shown in Table 11.

According to algorithm 3, the nonlinear programming model is constructed as follows:

$$
\begin{aligned}
& \min f=\rho_{12}+\delta_{12}+\rho_{13}+\delta_{13}+\rho_{23}+\delta_{23}, \\
& \operatorname{s.t}\left\{\begin{array}{l}
\frac{\omega_{1}}{\omega_{1}+\omega_{2}}+\rho_{12}-0.79 \geq 0, \\
\frac{\omega_{1}}{\omega_{1}+\omega_{3}}+\rho_{13}-0.667 \geq 0, \\
\frac{\omega_{2}}{\omega_{2}+\omega_{3}}+\rho_{23}-0.575 \geq 0, \\
\frac{\omega_{1}}{\omega_{1}+\omega_{2}}-\delta_{12}+0.107 \geq 0, \\
\frac{\omega_{1}}{\omega_{1}+\omega_{3}}-\delta_{13}+0.25 \geq 0, \\
\frac{\omega_{2}}{\omega_{2}+\omega_{3}}-\delta_{23}+0.323 \geq 0, \\
\omega_{1}+\omega_{2}+\omega_{3}=1, \\
0 \leq \omega_{1} \leq 1,0 \leq \omega_{2} \leq 1,0 \leq \omega_{3} \leq 1, \\
\rho_{12} \geq 0, \rho_{13} \geq 0, \rho_{23} \geq 0, \\
\delta_{12} \geq 0, \delta_{13} \geq 0, \delta_{23} \geq 0, \\
\rho_{12} * \delta_{12}=0, \rho_{13} * \delta_{13}=0, \rho_{23} * \delta_{23}=0 .
\end{array}\right.
\end{aligned}
$$

In this paper, we use LINGO software to solve formulas (26) and (27) and then derive the weight of each influencing factor in Figure 3.

From Figure 3, we can see $\omega_{1}=0.684, \omega_{2}=0.182$, and $\omega_{3}=0.134 ;$ in turn, the corresponding is $\omega_{S}=0.684$, $\omega_{O}=0.182$, and $\omega_{d}=0.134$.

According to formula (25) and the operation rules of IFNs, such as the final score calculation in $\mathrm{FM}_{1}$, the score after the weight of comprehensive influencing factors is calculated first, and the calculation formula is as follows: 
TABLE 2: Failure mode analysis table.

\begin{tabular}{|c|c|c|c|}
\hline No. & Failure mode & Failure effect & Failure reason \\
\hline F1 & $\begin{array}{l}\text { Poor insulation } \\
\text { The strength of the spool is not } \\
\text { enough, and the product splits after } \\
\text { winding }\end{array}$ & Magnetic flux leakage products & $\begin{array}{l}\text { Insufficient insulation level of material } \\
\text { (i)Low strength of plastic materials } \\
\text { (ii)Improper design of spool } \\
\text { (iii)Excessive tension design }\end{array}$ \\
\hline F3 & $\begin{array}{l}\text { Short circuit/open circuit of product } \\
\text { after power on and heating }\end{array}$ & $\begin{array}{l}\text { Cannot drive the action of customer } \\
\text { organization, customer cannot use it }\end{array}$ & Insufficient temperature resistance of materials \\
\hline $\mathrm{F} 4$ & Insufficient strength of end foot & Affect customer installation & Improper soldering and assembly design \\
\hline F5 & Poor surface roughness & Affect product life & Improper design of dimensional tolerance \\
\hline F6 & Poor concentricity & $\begin{array}{l}\text { Customer unable to install or not } \\
\text { suitable for installation }\end{array}$ & Improper design of dimensional tolerance \\
\hline F7 & $\begin{array}{l}\text { The magnetic force of the product is } \\
\text { insufficient after being electrified }\end{array}$ & $\begin{array}{l}\text { Unable to drive customer } \\
\text { organization action }\end{array}$ & $\begin{array}{l}\text { (i)The magnetic permeability of the material is } \\
\text { insufficient, and the attraction is insufficient after } \\
\text { being electrified } \\
\text { (ii)Improper design }\end{array}$ \\
\hline F8 & Residual magnetic field after power on & $\begin{array}{l}\text { Unable to drive customer } \\
\text { organization action }\end{array}$ & $\begin{array}{l}\text { (i)The magnetic permeability of the material is } \\
\text { insufficient, and the attraction is insufficient after } \\
\text { being electrified } \\
\text { (ii)The thickness of the lower wall is thin }\end{array}$ \\
\hline F9 & Appearance corrosion and rust & $\begin{array}{l}\text { Poor appearance, customer } \\
\text { dissatisfied }\end{array}$ & $\begin{array}{c}\text { The antirust layer is too thin and the process is } \\
\text { improper }\end{array}$ \\
\hline
\end{tabular}

TABLE 3: $S$ assessment criteria.

\begin{tabular}{|c|c|c|}
\hline Influence & Assessment criteria: severity of consequences (customer impact) & Grade \\
\hline $\begin{array}{l}\text { Failure of laws and regulations affecting safety or } \\
\text { government }\end{array}$ & $\begin{array}{c}\text { Work safety is affected or government regulations are not met without any } \\
\text { warning } \\
\text { In case of warning, it will affect work safety or not comply with government } \\
\text { regulations }\end{array}$ & $\mathrm{DH}$ \\
\hline Loss or reduction of expected function & $\begin{array}{l}\text { Loss of basic function (electromagnet does not work and does not affect safety) } \\
\text { Expected function reduction (electromagnet can work, performance level is } \\
\text { reduced) }\end{array}$ & $\begin{array}{l}\mathrm{VH} \\
\mathrm{H}\end{array}$ \\
\hline Loss or reduction of secondary function & $\begin{array}{l}\text { Loss of secondary function (failure of comfort and convenience function) } \\
\text { Lower secondary function (lower comfort and convenience function level) }\end{array}$ & $\begin{array}{c}\mathrm{MH} \\
\mathrm{M}\end{array}$ \\
\hline Customer feedback & $\begin{array}{l}\text { Electromagnets work, and most customers }(>75 \%) \text { perceive noise and } \\
\text { appearance } \\
\text { Electromagnets work, and some customers }(50 \%) \text { perceive noise and appearance } \\
\text { The electromagnet works, and a small number of customers }(<25 \%) \text { are aware of } \\
\text { noise and appearance }\end{array}$ & $\begin{array}{l}\text { ML } \\
\mathrm{L} \\
\mathrm{VL}\end{array}$ \\
\hline No impact & No detectable impact & EL \\
\hline
\end{tabular}

TABle 4: $O$ assessment criteria.

\begin{tabular}{lcc}
\hline Possibility of failure & Evaluation criteria: the possibility of causes & Grade \\
\hline Very high & New technology and new design without corresponding history & DH \\
\hline High & $\begin{array}{c}\text { Inevitable failure due to new design, new application, or change of operating conditions } \\
\text { New design, new application, or change of operating conditions may lead to failure } \\
\text { Uncertain failures due to new design, new application, or change of operating conditions }\end{array}$ & $\begin{array}{c}\text { EH } \\
\text { VH } \\
\text { H }\end{array}$ \\
\hline \multirow{2}{*}{ Medium } & Failure of similar design and design test & MH \\
& Occasional failures in design and testing & M \\
\hline Low & Isolated failures in similar designs and design tests & ML \\
\hline Very low & Almost identical designs and isolated failures in design testing & VL \\
\hline
\end{tabular}


TABle 5: $D$ assessment criteria.

\begin{tabular}{|c|c|c|}
\hline $\begin{array}{l}\text { Opportunity } \\
\text { discovery }\end{array}$ & Evaluation criteria: possibility of discovery through design control & Grade \\
\hline Very very low & There is no current design control that cannot be found or analyzed & EL \\
\hline Very low & Design analysis has weak discovery ability & VL \\
\hline Low & $\begin{array}{l}\text { Verification of a product that has passed testing (subsystem or system testing, e.g., noise and shipping) prior } \\
\text { to release }\end{array}$ & $\mathrm{L}$ \\
\hline Medium low & Verification of products using fault testing before product release (testing of subsystem or system failure) & ML \\
\hline Medium & $\begin{array}{l}\text { Verification of products using failure testing before product release (durability testing subsystem or system, } \\
\text { such as function check) }\end{array}$ & $\mathrm{M}$ \\
\hline Medium high & Product validation by testing before design freezing & $\mathrm{MH}$ \\
\hline High & Product confirmation by fault test before design freezing & $\mathrm{H}$ \\
\hline Very high & Use old test to confirm products before design freezing & $\mathrm{VH}$ \\
\hline Very very high & Design data analysis has strong ability of discovery & $\mathrm{EH}$ \\
\hline Extremely high & FMs cannot occur because of a fully preventive design & $\mathrm{DH}$ \\
\hline
\end{tabular}

TABLE 6: Evaluation table of evaluators in natural language.

\begin{tabular}{|c|c|c|c|c|c|c|c|c|c|}
\hline \multirow{3}{*}{ Failure mode } & \multicolumn{9}{|c|}{ Evaluators } \\
\hline & \multicolumn{3}{|c|}{ E1 } & \multicolumn{3}{|c|}{ E2 } & \multicolumn{3}{|c|}{ E3 } \\
\hline & S & $\mathrm{O}$ & $\mathrm{D}$ & S & $\mathrm{O}$ & $\mathrm{D}$ & S & $\mathrm{O}$ & $\mathrm{D}$ \\
\hline$\overline{\text { F1 }}$ & $\mathrm{VH}$ & EL & $\mathrm{VL}$ & $\mathrm{VH}$ & $\mathrm{L}$ & $\mathrm{VL}$ & $\mathrm{VH}$ & VL & $\overline{E L}$ \\
\hline $\mathrm{F} 2$ & $\mathrm{VH}$ & $\mathrm{L}$ & $\mathrm{EL}$ & $\mathrm{H}$ & $\mathrm{L}$ & VL & $\mathrm{H}$ & EL & VL \\
\hline F3 & $\mathrm{MH}$ & VL & ML & $\mathrm{VH}$ & $\mathrm{EL}$ & VL & $\mathrm{VH}$ & VL & VL \\
\hline $\mathrm{F} 4$ & $\mathrm{MH}$ & $\mathrm{L}$ & EL & $\mathrm{VH}$ & $\mathrm{VL}$ & M & VH & $\mathrm{L}$ & EL \\
\hline F5 & M & VL & ML & $\mathrm{MH}$ & $\mathrm{L}$ & EL & $\mathrm{MH}$ & $\mathrm{L}$ & $\mathrm{L}$ \\
\hline F6 & $\mathrm{EH}$ & EL & $\mathrm{VL}$ & $\mathrm{EH}$ & $\mathrm{L}$ & $\mathrm{M}$ & $\mathrm{EH}$ & EL & $\mathrm{EL}$ \\
\hline F7 & $\mathrm{VH}$ & $\mathrm{L}$ & VL & $\mathrm{MH}$ & $\mathrm{M}$ & $\mathrm{L}$ & $\mathrm{MH}$ & $\mathrm{L}$ & $\mathrm{L}$ \\
\hline F8 & $\mathrm{MH}$ & $\mathrm{M}$ & $\mathrm{L}$ & $\mathrm{VH}$ & EL & $\mathrm{EL}$ & $\mathrm{H}$ & VL & VL \\
\hline F9 & $\mathrm{M}$ & VL & $\mathrm{VL}$ & $\mathrm{M}$ & $\mathrm{VL}$ & $\mathrm{VL}$ & $\mathrm{M}$ & VVL & VL \\
\hline
\end{tabular}

TABLE 7: Evaluation table under IFN.

\begin{tabular}{|c|c|c|c|c|c|c|c|c|c|c|c|c|c|c|c|c|c|c|}
\hline \multirow{4}{*}{$\begin{array}{l}\text { Failure mode } \\
\text { F1 }\end{array}$} & \multicolumn{18}{|c|}{ Evaluators } \\
\hline & \multicolumn{6}{|c|}{ E1 } & \multicolumn{6}{|c|}{ E2 } & \multicolumn{6}{|c|}{ E3 } \\
\hline & \multicolumn{2}{|c|}{$S$} & \multicolumn{2}{|c|}{$\mathrm{O}$} & \multicolumn{2}{|c|}{$\mathrm{D}$} & \multicolumn{2}{|c|}{$S$} & \multicolumn{2}{|c|}{$\mathrm{O}$} & \multicolumn{2}{|c|}{$\mathrm{D}$} & \multicolumn{2}{|c|}{ S } & \multicolumn{2}{|c|}{$\mathrm{O}$} & \multicolumn{2}{|c|}{$\mathrm{D}$} \\
\hline & 0.8 & 0.1 & 0.1 & 0.75 & 0.1 & 0.75 & 0.8 & 0.1 & 0.25 & 0.6 & 0.1 & 0.75 & 0.8 & 0.1 & 0.1 & 0.75 & 0.1 & 0.9 \\
\hline $\mathrm{F} 2$ & 0.8 & 0.1 & 0.25 & 0.6 & 0.1 & 0.9 & 0.7 & 0.2 & 0.25 & 0.6 & 0.1 & 0.75 & 0.7 & 0.2 & 0.1 & 0.75 & 0.1 & 0.75 \\
\hline F3 & 0.6 & 0.3 & 0.1 & 0.75 & 0.5 & 0.5 & 0.8 & 0.1 & 0.1 & 0.9 & 0.1 & 0.75 & 0.8 & 0.1 & 0.1 & 0.75 & 0.1 & 0.75 \\
\hline $\mathrm{F} 4$ & 0.6 & 0.3 & 0.25 & 0.6 & 0.1 & 0.9 & 0.8 & 0.1 & 0.1 & 0.75 & 0.5 & 0.5 & 0.8 & 0.1 & 0.25 & 0.6 & 0.1 & 0.9 \\
\hline F5 & 0.5 & 0.5 & 0.1 & 0.75 & 0.4 & 0.5 & 0.6 & 0.3 & 0.25 & 0.6 & 0.1 & 0.9 & 0.6 & 0.3 & 0.25 & 0.6 & 0.25 & 0.6 \\
\hline F6 & 0.9 & 0.1 & 0.1 & 0.9 & 0.1 & 0.75 & 0.9 & 0.1 & 0.5 & 0.5 & 0.5 & 0.5 & 0.9 & 0.1 & 0.1 & 0.9 & 0.1 & 0.9 \\
\hline F7 & 0.8 & 0.1 & 0.25 & 0.6 & 0.1 & 0.75 & 0.6 & 0.3 & 0.25 & 0.6 & 0.25 & 0.6 & 0.6 & 0.3 & 0.25 & 0.6 & 0.25 & 0.6 \\
\hline F8 & 0.6 & 0.3 & 0.5 & 0.5 & 0.1 & 0.9 & 0.8 & 0.1 & 0.1 & 0.9 & 0.1 & 0.9 & 0.7 & 0.2 & 0.1 & 0.75 & 0.1 & 0.75 \\
\hline F9 & 0.5 & 0.5 & 0.1 & 0.75 & 0.1 & 0.75 & 0.5 & 0.5 & 0.1 & 0.75 & 0.1 & 0.75 & 0.5 & 0.5 & 0.1 & 0.9 & 0.1 & 0.75 \\
\hline
\end{tabular}

TABle 8: Comparison of influencing factors.

\begin{tabular}{lccccccccccc}
\hline & \multicolumn{1}{c}{ Evaluator } & E1 & \multicolumn{1}{c}{ Evaluator E2 } & \multicolumn{3}{c}{ Evaluator E3 } \\
\hline $\mathrm{O}$ & $\mathrm{S}$ & $\mathrm{O}$ & $\mathrm{D}$ & $\mathrm{O}$ & $\mathrm{S}$ & $\mathrm{O}$ & $\mathrm{D}$ & $\mathrm{O}$ & $\mathrm{S}$ & $\mathrm{O}$ & $\mathrm{D}$ \\
$\mathrm{S}$ & $\mathrm{M}$ & $\mathrm{H}$ & $\mathrm{MH}$ & $\mathrm{S}$ & $\mathrm{M}$ & $\mathrm{VH}$ & $\mathrm{M}$ & $\mathrm{S}$ & $\mathrm{M}$ & $\mathrm{VH}$ & $\mathrm{H}$ \\
$\mathrm{O}$ & $\mathrm{H}^{\prime}$ & $\mathrm{M}$ & $\mathrm{ML}$ & $\mathrm{O}$ & $\mathrm{VH}^{\prime}$ & $\mathrm{M}$ & $\mathrm{M}$ & $\mathrm{O}$ & $\mathrm{VH}^{\prime}$ & $\mathrm{M}$ & $\mathrm{H}$ \\
$\mathrm{D}$ & $\mathrm{MH}^{\prime}$ & $\mathrm{ML}^{\prime}$ & $\mathrm{M}$ & $\mathrm{D}$ & $\mathrm{M}^{\prime}$ & $\mathrm{M}^{\prime}$ & $\mathrm{M}$ & $\mathrm{D}$ & $\mathrm{H}^{\prime}$ & $\mathrm{H}^{\prime}$ & $\mathrm{M}$ \\
\hline
\end{tabular}

Here, $\mathrm{H}^{\prime}$ is the complement of $\mathrm{H}$ natural language. For example, the IFN corresponding to $\mathrm{H}$ natural language is $(0.70,0.20)$, and then the IFN corresponding to $\mathrm{H}^{\prime}$ natural language is $(0.20,0.70)$. 
TABLe 9: Perfect product consistency intuitionistic fuzzy matrix for every evaluator.

\begin{tabular}{|c|c|c|c|c|c|c|}
\hline \multicolumn{7}{|c|}{ Evaluator E1 } \\
\hline & \multicolumn{2}{|c|}{ S } & \multicolumn{2}{|c|}{$\mathrm{O}$} & \multicolumn{2}{|c|}{$\mathrm{D}$} \\
\hline S & 0.5 & 0.5 & 0.7 & 0.2 & 0.667 & 0.25 \\
\hline $\mathrm{O}$ & 0.2 & 0.7 & 0.5 & 0.5 & 0.4 & 0.5 \\
\hline $\mathrm{D}$ & 0.25 & 0.667 & 0.5 & 0.4 & 0.5 & 0.5 \\
\hline \multicolumn{7}{|c|}{ Evaluator E2 } \\
\hline & \multicolumn{2}{|c|}{$\mathrm{S}$} & \multicolumn{2}{|c|}{$\mathrm{O}$} & \multicolumn{2}{|c|}{$\mathrm{D}$} \\
\hline S & 0.5 & 0.5 & 0.8 & 0.1 & 0.667 & 0.25 \\
\hline $\mathrm{O}$ & 0.1 & 0.8 & 0.5 & 0.5 & 0.5 & 0.5 \\
\hline $\mathrm{D}$ & 0.25 & 0.667 & 0.5 & 0.5 & 0.5 & 0.5 \\
\hline \multicolumn{7}{|c|}{ Evaluator E3 } \\
\hline & \multicolumn{2}{|c|}{ S } & \multicolumn{2}{|c|}{$\mathrm{O}$} & \multicolumn{2}{|c|}{$\mathrm{D}$} \\
\hline S & 0.5 & 0.5 & 0.8 & 0.1 & 0.667 & 0.25 \\
\hline $\mathrm{O}$ & 0.1 & 0.8 & 0.5 & 0.5 & 0.7 & 0.2 \\
\hline $\mathrm{D}$ & 0.25 & 0.667 & 0.2 & 0.7 & 0.5 & 0.5 \\
\hline
\end{tabular}

TABLE 10: Evaluation information of influencing factors after aggregation.

\begin{tabular}{|c|c|c|c|c|c|c|}
\hline \multirow[b]{2}{*}{$S$} & \multicolumn{2}{|c|}{$S$} & \multicolumn{2}{|c|}{$\mathrm{O}$} & \multicolumn{2}{|c|}{$\mathrm{D}$} \\
\hline & 0.5000 & 0.5000 & 0.7669 & 0.1245 & 0.6670 & 0.2500 \\
\hline $\mathrm{O}$ & 0.1245 & 0.7669 & 0.5000 & 0.5000 & 0.5231 & 0.3648 \\
\hline $\mathrm{D}$ & 0.2500 & 0.6670 & 0.3648 & 0.5231 & 0.5000 & 0.5000 \\
\hline
\end{tabular}

TABLE 11: Aggregation matrix after iterative operation.

\begin{tabular}{|c|c|c|c|c|c|c|}
\hline & \multicolumn{2}{|c|}{$S$} & \multicolumn{2}{|c|}{$\mathrm{O}$} & \multicolumn{2}{|c|}{$\mathrm{D}$} \\
\hline$S$ & 0.500 & 0.500 & 0.790 & 0.107 & 0.667 & 0.250 \\
\hline $\mathrm{O}$ & 0.107 & 0.790 & 0.500 & 0.500 & 0.575 & 0.323 \\
\hline $\mathrm{D}$ & 0.250 & 0.667 & 0.323 & 0.575 & 0.500 & 0.500 \\
\hline
\end{tabular}

Local optimal solution found.

Objective value:

Infeasibilities:

Total solver iterations:

Elapsed runtime seconds:

Model Class:

Total variables:

Nonlinear variables:

Integer variables:

Total constraints:

Nonlinear constraints:

Total nonzeros:

Nonlinear nonzeros:
$0.8578657 \mathrm{E}-01$

0.000000

7

1.93

NLP

\begin{tabular}{cc} 
Variable & Value \\
A12 & 0.000000 \\
B12 & 0.000000 \\
A13 & 0.000000 \\
B13 & $0.8578657 \mathrm{E}-01$ \\
A23 & 0.000000 \\
B23 & 0.000000 \\
\hline W1 & 0.6838540 \\
W2 & 0.1817840 \\
W3 & 0.1343621 \\
\hline
\end{tabular}

Reduced Cost

0.1727102

1.000000

1.000000

0.000000

0.4383739

1.000000

0.000000

0.000000

0.000000

Figure 3: Influencing factor weight derivation chart. 
TABLE 12: Summary of final results.

\begin{tabular}{llc}
\hline Failure mode & Score & Sort \\
\hline F1 & 6.658 & 9 \\
F2 & 6.812 & 8 \\
F3 & 7.018 & 6 \\
F4 & 7.299 & 5 \\
F5 & 7.664 & 2 \\
F6 & 6.943 & 7 \\
F7 & 7.482 & 4 \\
F8 & 7.497 & 3 \\
F9 & 8.011 & 1 \\
\hline
\end{tabular}

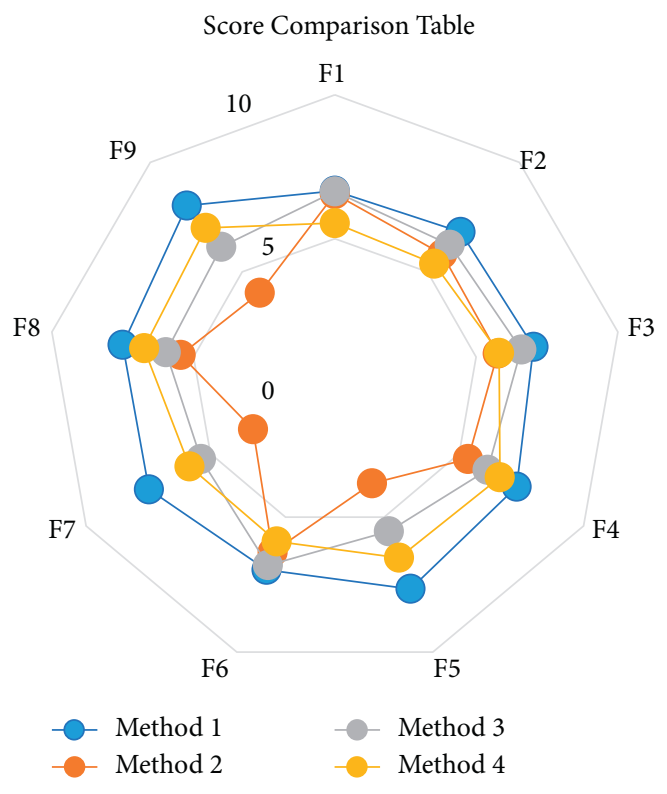

Figure 4: Comparison score table.

$$
\begin{aligned}
& 0.684 *(0.9,0.1)+0.684 *(0.8,0.1)+0.684 *(0.8,0.1)+0.182 *(0.1,0.8)+0.182 *(0.1,0.9)+0.182 *(0.1,0.8) \\
& \quad+0.134 *(0.1,0.9)+0.134 *(0.1,0.8)+0.134 *(0.1,0.9)=6.658
\end{aligned}
$$

Similarly, the final scores for other FMs are calculated and ranked according to the final scores, as shown in Table 12.

5.2. Discussions. This approved method is compared with the other three methods in terms of final scores. The three methods for comparison are traditional FMEA, only calculating the weight of influencing factors, and only calculating the weight of evaluating personnel. Except for the weight, the other steps are the same as the new method. The score table is shown in Figure 4.

It is obvious from the two tables that there is a big difference between the traditional FMEA ranking and the score of this new method. This is because the traditional FMEA does not specify the weight of influencing factors and the weight of evaluation members; so, when the score function is used to calculate the score, the value of evaluation content is all involved in the calculation. However, from the above calculation, the result is $\omega_{S}=0.684, \omega_{O}=0.182$, $\omega_{d}=0.134, \omega_{E 1}=0.316, \omega_{E 2}=0.34, \omega_{E 3}=0.344$. According to the operation rules of IFNs, the rule calculation is carried out twice, and the proportion of each calculation is the weight. On the other hand, the calculated results are quite different from the default weight, especially the weight of influencing factors, so there will be a lot of differences in scores. If we evaluate according to the 10-point system, the final score difference may be more crucial.

\section{Conclusions}

To avoid the disadvantages of traditional FMEA, this paper presents IFN to reflect the final ranking of evaluation team for electromagnet in manufacturing systems. The failure evaluation mode is a general and comprehensive decision of the consequences and effects of failure. Based on the distinguishing features of subordination degree and nonsubordination degree of IFN, the error of final calculation failure mode score can be reduced to a more manageable 
level. In addition, this paper supplements the incomplete determination of weight information with traditional FMEA and calculates the best evaluation information by using the concept of similarity measure.

On the other hand, in view of the unknown weight of influencing factors, this paper compares and evaluates influencing factors by many evaluators forming a contrast matrix. A nonlinear programming model based on the contrast matrix of influencing factors was built to simulate the evaluation results of the weight of influencing factors. Then, the absolute distance between each evaluator's decision information and the best decision information are calculated in two different ways. The contributions of this paper lie in that we put forward a potential FMEA method of electromagnet based on IFN in empirical study. This paper also discusses the further improving measures of IFN and presents future research directions for other mechanical and electrical products.

\section{Data Availability}

The data of this paper come from the practice and experimental design of enterprises.

\section{Conflicts of Interest}

The authors declare that they have no conflicts of interest.

\section{Acknowledgments}

This work was supported by the National Natural Science Foundation of China (nos. 72071149 and 71671130) and Provincial Natural Science Foundation, Zhejiang, China (no. LY20G010014).

\section{References}

[1] M. Giannakis and T. Papadopoulos, "Supply chain sustainability: a risk management approach," International Journal of Production Economics, vol. 171, pp. 455-470, 2016.

[2] Y. Geum, Y. Cho, and Y. Park, "A systematic approach for diagnosing service failure: service-specific FMEA and grey relational analysis approach," Mathematical and Computer Modelling, vol. 54, no. 11-12, pp. 3126-3142, 2011.

[3] M. S. Kirkire, S. B. Rane, and J. R. Jadhav, "Risk management in medical product development process using traditional FMEA and fuzzy linguistic approach: a case study," Journal of Industrial Engineering International, vol. 11, no. 4, pp. 595611, 2015.

[4] S. Dorosti, M. Fathi, S. J. Ghoushchi et al., "Patient waiting time management through fuzzy based failure mode and effect analysis," Journal of Intelligent and Fuzzy Systems, vol. 38, no. 6, pp. 1-12, 2020.

[5] W. Huang, Y. Li, X. Kou et al., "Using a FMEA-TIFIAD approach to identify the risk of railway dangerous goods transportation system," Group Decision and Negotiation, vol. 10, no. 10, pp. 1-33, 2020.

[6] H.-C. Liu, L. Liu, and N. Liu, "Risk evaluation approaches in failure mode and effects analysis: a literature review," Expert Systems with Applications, vol. 40, no. 2, pp. 828-838, 2013.

[7] S.-S. He, Y.-T. Wang, J.-J. Peng, and J.-Q. Wang, "Risk ranking of wind turbine systems through an improved FMEA based on probabilistic linguistic information and the TODIM method," Journal of the Operational Research Society, vol. 4, pp. 1-14, 2021.

[8] M. D. Ramere and O. T. Laseinde, "Optimization of condition-based maintenance strategy prediction for aging automotive industrial equipment using FMEA," Procedia Computer Science, vol. 180, no. 16, pp. 229-238, 2021.

[9] H.-W. Lo, J. J. H. Liou, C.-N. Huang, and Y.-C. Chuang, "A novel failure mode and effect analysis model for machine tool risk analysis," Reliability Engineering \& System Safety, vol. 183, pp. 173-183, 2019.

[10] Y.-X. Xue, J.-X. You, X.-D. Lai, and H.-C. Liu, “An intervalvalued intuitionistic fuzzy MABAC approach for material selection with incomplete weight information," Applied Soft Computing, vol. 38, pp. 703-713, 2016.

[11] J. Qin, Y. Xi, and W. Pedrycz, "Failure mode and effects analysis (FMEA) for risk assessment based on interval type-2 fuzzy evidential reasoning method," Applied Soft Computing, vol. 89, pp. 106-134, 2020.

[12] H.-C. Liu, J.-X. You, and C.-Y. Duan, "An integrated approach for failure mode and effect analysis under intervalvalued intuitionistic fuzzy environment," International Journal of Production Economics, vol. 207, pp. 163-172, 2019.

[13] S. Daneshvar, M. Yazdi, and K. A. Adesina, "Fuzzy smart failure modes and effects analysis to improve safety performance of system: case study of an aircraft landing system," Quality and Reliability Engineering International, vol. 36, no. 3, pp. 890-909, 2020.

[14] Y. Mohammad, "Improving failure mode and effect analysis (FMEA) with consideration of uncertainty handling as an interactive approach," International Journal on Interactive Design and Manufacturing, vol. 13, pp. 441-458, 2018.

[15] Z. Liu and F. Xiao, "An intuitionistic evidential method for weight determination in FMEA based on belief entropy," Entropy, vol. 21, no. 3, pp. 211-227, 2019.

[16] W. Wang, X. Liu, X. Chen, and Y. Qin, "Risk assessment based on hybrid FMEA framework by considering decision maker's psychological behavior character," Computers \& Industrial Engineering, vol. 136, pp. 516-527, 2019.

[17] H. Zhang, Y. Dong, I. Palomares-Carrascosa et al., "Failure mode and effect analysis in a linguistic context: a consensusbased multiattribute group decision-making approach," IEEE Transactions on Reliability, vol. 99, pp. 1-17, 2018.

[18] H. Zheng and Y. Tang, "A novel failure mode and effects analysis model using triangular distribution-based basic probability assignment in the evidence theory," IEEE Access, vol. 8, pp. 66813-66827, 2020.

[19] S. Boral, S. K. Chaturvedi, V. N. A. Naikan et al., "An integrated approach for fuzzy failure modes and effects analysis using fuzzy AHP and fuzzy MAIRCA," Engineering Failure Analysis, vol. 108, pp. 104195-104211, 2019.

[20] Z.-p. Tian, J.-q. Wang, and H.-y. Zhang, “An integrated approach for failure mode and effects analysis based on fuzzy best-worst, relative entropy, and VIKOR methods," Applied Soft Computing, vol. 72, pp. 636-646, 2018.

[21] A. M. Talib, "Fuzzy VIKOR Approach to evaluate the information security policies and analyze the content of press agencies in gulf countries," Journal of Information Security, vol. 11, no. 4, pp. 189-200, 2020.

[22] L. Li and W. Yue, "Dynamic uncertain causality graph based on Intuitionistic fuzzy sets and its application to root cause analysis," Applied Intelligence, vol. 50, no. 1, pp. 241-255, 2020.

[23] X. Zhang and Z. Xu, "Soft computing based on maximizing consensus and fuzzy TOPSIS approach to interval-valued 
intuitionistic fuzzy group decision making," Applied Soft Computing, vol. 26, pp. 42-56, 2015.

[24] Z. Yue, "Application of the projection method to determine weights of decision makers for group decision making," Scientia Iranica, vol. 19, no. 3, pp. 872-878, 2012.

[25] F. L. Gaol and T. Matsuo, "The simulation of implications of sensor technology on the new product development to solve lot-sizing problems with fuzzy approach," Journal of Sensors, vol. 2020, Article ID 3503895, 15 pages, 2020. 